\begin{tabular}{|c|l|}
\hline Title & Identification and Herc5-mediated ISGylation of novel target proteins \\
\hline Author(s) & Takeuchi, Tomoharu; Inoue, Satoshi; Y okosawa, Hidey oshi \\
\hline Citation & $\begin{array}{l}\text { Biochemical and Biophysical Research Communications, 348(2), 473-477 } \\
\text { https://doi.org/40.1016/.bbrc.2006.07.076 }\end{array}$ \\
\hline Issue Date & $2006-09-22$ \\
\hline Doc URL & http://hdl.handle.net/2115/14737 \\
\hline Type & article (author version) \\
\hline File Information & BBRC 348 473 2006.pdf \\
\hline
\end{tabular}

Instructions for use 


\section{Identification and Herc5-mediated ISGylation of novel target proteins}

Tomoharu Takeuchi $^{1}$, Satoshi Inoue ${ }^{2}$, Hideyoshi Yokosawa ${ }^{1^{*}}$

${ }^{1}$ Department of Biochemistry, Graduate School of Pharmaceutical Sciences, Hokkaido

University, Sapporo 060-0812, Japan

${ }^{2}$ Department of Geriatric Medicine, Graduate School of Medicine, The University of Tokyo,

7-3-1 Hongo, Bunkyo-ku, Tokyo 113-8655, Japan

*Corresponding author. Fax: +81 117064900

E-mail address: yoko@pharm.hokudai.ac.jp (H. Yokosawa)

Send correspondence to:

Hideyoshi Yokosawa, Ph.D., Department of Biochemistry, Graduate School of Pharmaceutical Sciences, Hokkaido University, Sapporo 060-0812, Japan

Tel.: 81-11-706-3754; Fax: 81-11-706-4900. E-mail: yoko@pharm.hokudai.ac.jp 


\section{Abstract}

ISG15, a protein containing two ubiquitin-like domains, is an interferon-stimulated gene product that functions in antiviral response and is conjugated to various cellular proteins (ISGylation) upon interferon stimulation. ISGylation occurs via a pathway similar to the pathway for ubiquitination that requires the sequential action of E1/E2/E3: The E1 (UBE1L), E2 (UbcH8), and E3 (Efp/Herc5) enzymes for ISGylation have been hitherto identified. In this study, we identified six novel candidate target proteins for ISGylation by a proteomic approach. Four candidate target proteins were demonstrated to be ISGylated in UBE1L- and UbcH8-dependent manners, and ISGylation of the respective target proteins was stimulated by Herc5. In addition, Herc5 was capable of binding with the respective target proteins. Thus, these results suggest that Herc5 functions as a general E3 ligase for protein ISGylation.

Keywords: ISG15; interferon; ubiquitin; Herc5

Abbreviations: ISG, interferon-stimulated gene; E1, ubiquitin-activating enzyme; E2, ubiquitin-conjugating enzyme; E3, ubiquitin ligase; XP, xeroderma pigmentosum; ERCC, excision-repair, complementing defective, in Chinese hamster; STK, serine/threonine kinase; RGS, regulator of G protein signaling; PP, protein phosphatase; Efp, estrogen-responsive finger protein; Herc, HECT domain and RCC1-like domain containing protein. 


\section{Introduction}

Type I interferon functions in cellular antiviral response via induction of genes called interferon-stimulated genes (ISGs) [1]. Recently, ISG15, one of the ISGs, was found to function as an antiviral protein against Sindbis virus and HIV-1 [2,3] although the molecular mechanism remains unknown. ISG15 contains two ubiquitin-like domains and belongs to the ubiquitin-like protein family. The expression of ISG15 is induced by interferon stimulation and ISG15 is conjugated to various cellular proteins (ISGylation) in a manner similar to ubiquitination that is catalyzed by the sequential action of E1 (ubiquitin-activating enzyme), E2 (ubiquitin-conjugating enzyme) and E3 (ubiquitin ligase) [4,5]. Target proteins modified with ISG15 [6-8], the E1 (UBE1L) and E2 (UbcH8) enzymes functioning in ISGylation [9-11], and a de-ISGylating enzyme (UBP43) [12] have been identified, but biological consequences of ISGylation have been studied in only a few cases [13-15]. Recently, Efp and Herc5 have been reported to function as E3 ligases for ISGylation [16,17], but there seems to be a difference in function between Efp and Herc5 because Herc5, but not Efp, influences the ISGylation status of whole cellular proteins [16,17].

In this study, we identified six novel candidate target proteins for ISGylation and confirmed that four identified proteins, XPD (ERCC2), STK38, RGS3 isoform 1 and $\alpha$-tubulin, are actually ISGylated in UBE1L- and UbcH8-dependent manners. In addition, we found that Herc5 is capable of binding with and stimulating ISGylation of the respective 
novel target proteins.

\section{Materials and methods}

Cell culture and transfection. HeLa cells were cultured in Dulbecco's modified Eagle's medium (Sigma) supplemented with 10\% heat-inactivated calf serum (Hyclone). Transfection was performed according to the standard calcium precipitation protocol.

Plasmid construction. The mammalian expression plasmids of ISG15, UBE1L, and UbcH8 were generated as described previously [15]. The open-reading frames of human XPD (ERCC2), STK38, RGS3 isoform 1, $\alpha$-tubulin, and Herc5 were amplified by PCR. All constructs were verified by DNA sequencing. To generate the expression plasmids, the PCR fragments were subcloned into pCI-neo-3Flag and pCI-neo-2S vectors that had been generated by inserting oligonucleotides encoding three repeats of Flag-tag sequence and two repeats of S-peptide sequence, respectively, into the pCI-neo mammalian expression vector (Promega). To generate the expression plasmid of Efp, the open reading frame of human Efp was cut from the Myc-Efp plasmid that had been constructed as described previously [18] and was subcloned into the pCI-neo-2S vector.

Isolation of ISGylated proteins. HeLa cells that had been transiently transfected with Flag-tagged ISG15, S-tagged UBE1L and S-tagged UbcH8 expression plasmids and cultured for 36 h were washed with ice-cold phosphate-buffered saline and lysed with RIPA buffer 
containing 50 mM Tris-HCl, pH 7.5, 150 mM NaCl, 0.1\% SDS, 0.5\% sodium deoxycholate, 1\% Nonidet P-40, $1 \mathrm{mM}$ DTT, and $5 \mathrm{mM}$ N-ethylmaleimide. The cell lysate was sonicated for $3 \mathrm{~s}$ and the debris was removed by centrifugation. The resulting supernatant was incubated with anti-Flag M2 antibody-immobilized agarose (Sigma), and the resulting immunoprecipitate was washed three times with RIPA buffer, followed by washing three times with a buffer containing $20 \mathrm{mM}$ Tris-HCl, pH 7.5, $500 \mathrm{mM} \mathrm{NaCl,} \mathrm{0.2 \%} \mathrm{Nonidet} \mathrm{P-40,}$ and $10 \%$ glycerol. The materials bound to the beads were eluted with $3 \times$ Flag peptide (Sigma) (200 $\mu \mathrm{g} / \mathrm{ml})$ and subjected to acetone precipitation.

SDS-PAGE and Western blotting. Isolated ISGylated proteins were separated by SDS-PAGE and stained with Coomassie Brilliant Blue. Western blotting was performed as described previously [13].

Peptide mass fingerprinting. Peptide mass fingerprinting was performed as described previously [19] except for the use of MS-fit (http://prospector.ucsf.edu/ucsfhtml4.0/msfit.htm) for analysis of peptide mass fingerprints. Protein classification was made by searching the $\mathrm{OMIM}^{\mathrm{TM}}$ database (http://www.ncbi.nlm.nih.gov/entrez/query.fcgi?db=OMIM).

Immunoprecipitation and affinity purification. To analyze ISGylation, HeLa cells that had been transiently transfected with indicated plasmids and cultured for $24 \mathrm{~h}$ were lysed with buffer A containing $50 \mathrm{mM}$ Tris-HCl, pH 7.5, $150 \mathrm{mM} \mathrm{NaCl,} \mathrm{0.1 \%} \mathrm{SDS,} \mathrm{0.5 \%} \mathrm{sodium}$ 
deoxycholate, 1\% Nonidet P-40, $1 \mathrm{mM}$ phenylmethylsulfonyl fluoride, and $5 \mathrm{mM}$ N-ethylmaleimide, and the supernatant of the cell lysate was subjected to immunoprecipitation using anti-Flag tag antibody-immobilized agarose beads. The resulting immunoprecipitate was washed five times with buffer A and subjected to Western blotting. To analyze the binding capacity of Herc5 or Efp, HeLa cells that had been transiently transfected with indicated plasmids and cultured for 24 h were lysed with buffer B containing $20 \mathrm{mM}$ Tris-HCl, pH 7.5, $150 \mathrm{mM} \mathrm{NaCl,} \mathrm{0.2 \%} \mathrm{Nonidet} \mathrm{P-40,} \mathrm{10 \%} \mathrm{glycerol,} \mathrm{and} 1 \mathrm{mM}$ phenylmethylsulfonyl fluoride, and the supernatant of the cell lysate was subjected to affinity purification using S-protein-immobilized agarose beads (Novagen). The resulting precipitate was washed three times with buffer B and subjected to Western blotting.

\section{Results and discussion}

Identification of proteins modified with ISG15

To determine the physiological meanings of ISGylation, it is necessary to identify proteins that are modified with ISG15. We carried out a proteomic analysis of ISGylated proteins. Flag-tagged ISG15, S-tagged UBE1L, and S-tagged UbcH8 were expressed in HeLa cells, and ISGylated proteins were isolated from the cell extract by immunoprecipitation with anti-Flag tag antibody-immobilized agarose beads and subsequent elution with $3 \times$ Flag peptide. The isolated proteins were separated by SDS-PAGE and stained with Coomassie 
Brilliant Blue (Fig. 1). The separated protein bands were subjected to peptide mass fingerprinting and ten candidate target proteins for ISGylation were identified (Table 1). Among them, six proteins are novel candidate target proteins that have not been reported.

To confirm the above results of peptide mass fingerprinting, we cloned four novel candidate target proteins for ISGylation, XPD (ERCC2), STK38, RGS3 isoform 1 and $\alpha$-tubulin. The respective Flag-tagged candidate target proteins were expressed together with T7-tagged ISG15, S-tagged UBE1L, and S-tagged UbcH8 in HeLa cells, and the extracts of transfected cells were subjected to immunoprecipitation using anti-Flag tag antibody-immobilized agarose beads and then to Western blotting with anti-Flag tag and anti-T7 tag antibodies (Fig. 2). In either case, two bands with slower mobilities (open arrowheads) than that of the original one (a closed arrowhead) were detected in the presence of both UBE1L and UbcH8 by immunoblotting with anti-Flag tag antibody (left panel), and the bands with the same slower mobilities were also detected by immunoblotting with anti-T7 tag antibody (right panel). These results suggest that XPD (ERCC2), STK38, RGS3 isoform 1 , and $\alpha$-tubulin are actually ISGylated.

Herc5 stimulates ISGylation of novel target proteins

Efp and Herc5 have been reported to be E3 ligases for ISGylation [16, 17], although they also function as ubiquitin E3 ligases [18, 20]. We carried out experiments to determine which 
E3 ligase stimulates ISGylation of four target proteins newly identified in this study, XPD (ERCC2), STK38, RGS3 isoform 1 and $\alpha$-tubulin. First, we constructed mammalian expression plasmids of Efp and Herc5 and expressed them together with T7-tagged ISG15, S-tagged UBE1L, and S-tagged UbcH8 in HeLa cells. The extracts of transfected cells were subjected to Western blotting with anti-T7 tag and anti-S peptide antibodies (Fig. 3). Consistent with previous reports [16, 17], Herc5, but not Efp, stimulated ISGylation of the whole cellular proteins. Next, the above four Flag-tagged target proteins were expressed together with T7-tagged ISG15, S-tagged UBE1L, S-tagged UbcH8, and S-tagged Herc5 or Efp in HeLa cells, and the extracts of transfected cells were subjected to immunoprecipitation using anti-Flag tag antibody-immobilized agarose beads and then to Western blotting with anti-Flag tag and anti-T7 tag antibodies (Fig. 4). ISGylation of STK38 or RGS3 isoform 1 was strongly stimulated by co-expression of Herc5 and very weakly by Efp (see two bands indicated by open arrowheads in either case). In the case of XPD (ERCC2), an additional band due to an ISGylated form was detected when Herc5, but not Efp, was co-expressed (see one band indicated by an arrow). On the other hand, ISGylation of $\alpha$-tubulin was stimulated by Efp as well as by Herc5 (see two bands indicated by open arrowheads). These results suggest that Herc5 functions as an E3 ligase for ISGylation of all four target proteins.

Herc5 is capable of binding with novel target proteins for ISGylation 
It is well known that the ubiquitin E3 ligase recognizes or binds with target proteins for ubiquitination [4]. We next determined whether Herc5 is capable of binding with four target proteins, XPD (ERCC2), STK38, RGS3 isoform 1 and $\alpha$-tubulin. The respective Flag-tagged target proteins were expressed together with S-tagged Herc5 or Efp in HeLa cells, and the extracts of transfected cells were subjected to affinity purification using S-protein-immobilized agarose beads and then to Western blotting with anti-Flag tag and anti-S peptide antibodies (Fig. 5). Herc5 was found to bind with each of the four target proteins, while Efp was found to bind weakly only with XPD (ERCC2).

In conclusion, we identified six novel target proteins for ISGylation and found that ISGylation of XPD (ERCC2), STK38, RGS3 isoform 1 and $\alpha$-tubulin is catalyzed by three enzymes, UBE1L, UbcH8, and Herc5. These results agree well with results of a previous study showing that Herc5 is an accelerator of a broad range of ISG15 conjugation [17]. The construction of an in vitro ISGylation system containing recombinant Herc5 together with ISG15, UBE1L and UbcH8 will verify the speculation that Herc5 functions as a general E3 ligase for protein ISGylation.

\section{Acknowledgements}

This study was supported in part by grants-in-aid for scientific research from the Ministry of Education, Culture, Sports, Science and Technology of Japan. 


\section{References}

[1] D.V. Kalvakolanu, Alternate interferon signaling pathways, Pharmacol. Ther. 100 (2003) $1-29$.

[2] D.J. Lenschow, N.V. Giannakopoulos, L.J. Gunn, C. Johnston, A.K. O'Guin, R.E. Schmidt,

B. Levine, H.W. Virgin, Identification of interferon-stimulated gene 15 as an antiviral molecule during Sindbis virus infection in vivo, J. Virol. 79 (2005) 13974-13983.

[3] A. Okumura, G. Lu, I. Pitha-Rowe, P.M. Pitha, Innate antiviral response targets HIV-1 release by the induction of ubiquitin-like protein ISG15, Proc. Natl. Acad. Sci. USA 103 (2006) 1440-1445.

[4] C.M. Pickart, Mechanisms underlying ubiquitination, Annu. Rev. Biochem. 70 (2001) 503-533.

[5] C.T. Dao, D.E. Zhang, ISG15: a ubiquitin-like enigma, Front. Biosci. 10 (2005) 2701-2722.

[6] M.P. Malakhov, K.I. Kim, O.A. Malakhova, B.S. Jacobs, E.C. Borden, D.E. Zhang, High-throughput immunoblotting. Ubiquitiin-like protein ISG15 modifies key regulators of signal transduction, J. Biol. Chem. 278 (2003) 16608-16613.

[7] C. Zhao, C. Denison, J.M. Huibregtse, S. Gygi, R.M. Krug, Human ISG15 conjugation targets both IFN-induced and constitutively expressed proteins functioning in diverse cellular 
pathways, Proc. Natl. Acad. Sci. USA 102 (2005) 10200-10205.

[8] N.V. Giannakopoulos, J.K. Luo, V. Papov, W. Zou, D.J. Lenschow, B.S. Jacobs, E.C.

Borden, J. Li, H.W. Virgin, D.E. Zhang, Proteomic identification of proteins conjugated to ISG15 in mouse and human cells, Biochem. Biophys. Res. Commun. 336 (2005) 496-506.

[9] W. Yuan, R.M. Krug, Influenza B virus NS1 protein inhibits conjugation of the interferon (IFN)-induced ubiquitin-like ISG15 protein, EMBO J. 20 (2001) 362-371.

[10] C. Zhao, S.L. Beaudenon, M.L. Kelley, M.B. Waddell, W. Yuan, B.A. Schulman, J.M. Huibregtse, R.M. Krug, The UbcH8 ubiquitin E2 enzyme is also the E2 enzyme for ISG15, an IFN- $\alpha / \beta$-induced ubiquitin-like protein, Proc. Natl. Acad. Sci. USA 101 (2004) 7578-7582.

[11] K.I. Kim, N.V. Giannakopoulos, H.W. Virgin, D.E. Zhang, Interferon-inducible ubiquitin E2, Ubc8, is a conjugating enzyme for protein ISGylation, Mol. Cell. Biol. 24 (2004) 9592-9600.

[12] M.P. Malakhov, O.A. Malakhova, K.I. Kim, K.J. Ritchie, D.E. Zhang, UBP43 (USP18) specifically removes ISG15 from conjugated proteins, J. Biol. Chem. 277 (2002) 9976-9981. [13] T. Takeuchi, H. Yokosawa, ISG15 modification of Ubc13 suppresses its ubiquitin-conjugating activity, Biochem. Biophys. Res. Commun. 336 (2005) 9-13. [14] W. Zou, V. Papov, O. Malakhova, K.I. Kim, C. Dao, J. Li, D.E. Zhang, ISG15 modification of ubiquitin E2 Ubc13 disrupts its ability to form thioester bond with ubiquitin, 
Biochem. Biophys. Res. Commun. 336 (2005) 61-68.

[15] T. Takeuchi, S. Iwahara, Y. Saeki, H. Sasajima, H. Yokosawa, Link between the ubiquitin conjugation system and the ISG15 conjugation system: ISG15 conjugation to the UbcH6 ubiquitin E2 enzyme, J. Biochem. (Tokyo) 138 (2005) 711-719.

[16] W. Zou, D.E. Zhang, The interferon-inducible ubiquitin-protein isopeptide ligase (E3) EFP also functions as an ISG15 E3 ligase, J. Biol. Chem. 281 (2006) 3989-3994.

[17] A. Dastur, S. Beaudenon, M. Kelley, R.M. Krug, J.M. Huibregtse, Herc5, an interferon-induced HECT E3 enzyme, is required for conjugation of ISG15 in human cells, J. Biol. Chem. 281 (2006) 4334-4338.

[18] T. Urano, T. Saito, T. Tsukui, M. Fujita, T. Hosoi, M. Muramatsu, Y. Ouchi, S. Inoue, Efp targets 14-3-3 sigma for proteolysis and promotes breast tumour growth, Nature 417 (2002) 871-875.

[19] M. Yamakami, T. Yoshimori, H. Yokosawa, Tom1, a VHS domain-containing protein, interacts with tollip, ubiquitin, and clathrin, J. Biol. Chem. 278 (2003) 52865-52872.

[20] R. Kroismayr, U. Baranyi, C. Stehlik, A. Dorfleutner, B.R. Binder, J. Lipp, HERC5, a HECT E3 ubiquitin ligase tightly regulated in LPS activated endothelial cells, J. Cell. Sci. 117 (2004) 4749-4756. 


\section{Figure legends}

Fig. 1. Identification of proteins ISGylated. The extract of HeLa cells that had expressed Flag-tagged ISG15 together with S-tagged UBE1L and S-tagged UbcH8 was subjected to immunoprecipitation (IP) with anti-Flag tag antibody-immobilized agarose beads, and isolated ISGylated proteins were separated by SDS-PAGE and stained with Coomassie Brilliant Blue (CBB). The bands identified by peptide mass fingerprinting are indicated by arrows. M, molecular mass markers.

Fig. 2. Four candidate target proteins, XPD (ERCC2), STK38, RGS3 isoform 1 and $\alpha$-tubulin, are ISGylated in UBE1L- and UbcH8-dependent manners. The extract of HeLa cells that had expressed the respective Flag-tagged candidate proteins together with T7-tagged ISG15, S-tagged UBE1L and S-tagged UbcH8 as indicated was subjected to immunoprecipitation (IP) with anti-Flag tag antibody-immobilized agarose beads. The resulting immunoprecipitate was subjected to Western blotting with anti-Flag tag and anti-T7 tag antibodies. ISGylated forms of the candidate proteins are indicated by open arrowheads and the original forms are indicated by closed arrowheads.

Fig. 3. Herc5 stimulates ISGylation of the whole cellular proteins. The extract of HeLa cells that had been transiently transfected with indicated plasmids was subjected to Western 
blotting with anti-T7 tag and anti-S peptide antibodies.

Fig. 4. Effects of co-expressions of Herc5 and Efp on ISGylation of four target proteins, XPD (ERCC2), STK38, RGS3 isoform 1 and $\alpha$-tubulin. The extracts of HeLa cells that had been transiently transfected with indicated plasmids were subjected to immunoprecipitation (IP) with anti-Flag tag antibody-immobilized beads and then to Western blotting with anti-Flag tag and anti-T7 tag antibodies. ISGylated forms of the target proteins are indicated by open arrowheads and the original forms are indicated by closed arrowheads. An additional band due to ISGylated XPD (ERCC2) is indicated by an arrow.

Fig. 5. Herc5 is capable of binding with four target proteins, XPD (ERCC2), STK38, RGS3 isoform 1 and $\alpha$-tubulin. The extract of HeLa cells that had expressed the respective Flag-tagged target proteins together with S-tagged Herc5 or Efp as indicated was subjected to affinity purification using S-protein-immobilized agarose beads and then to Western blotting with anti-Flag tag and anti-S peptide antibodies. 
Fig. 1. Takeuchi et al.

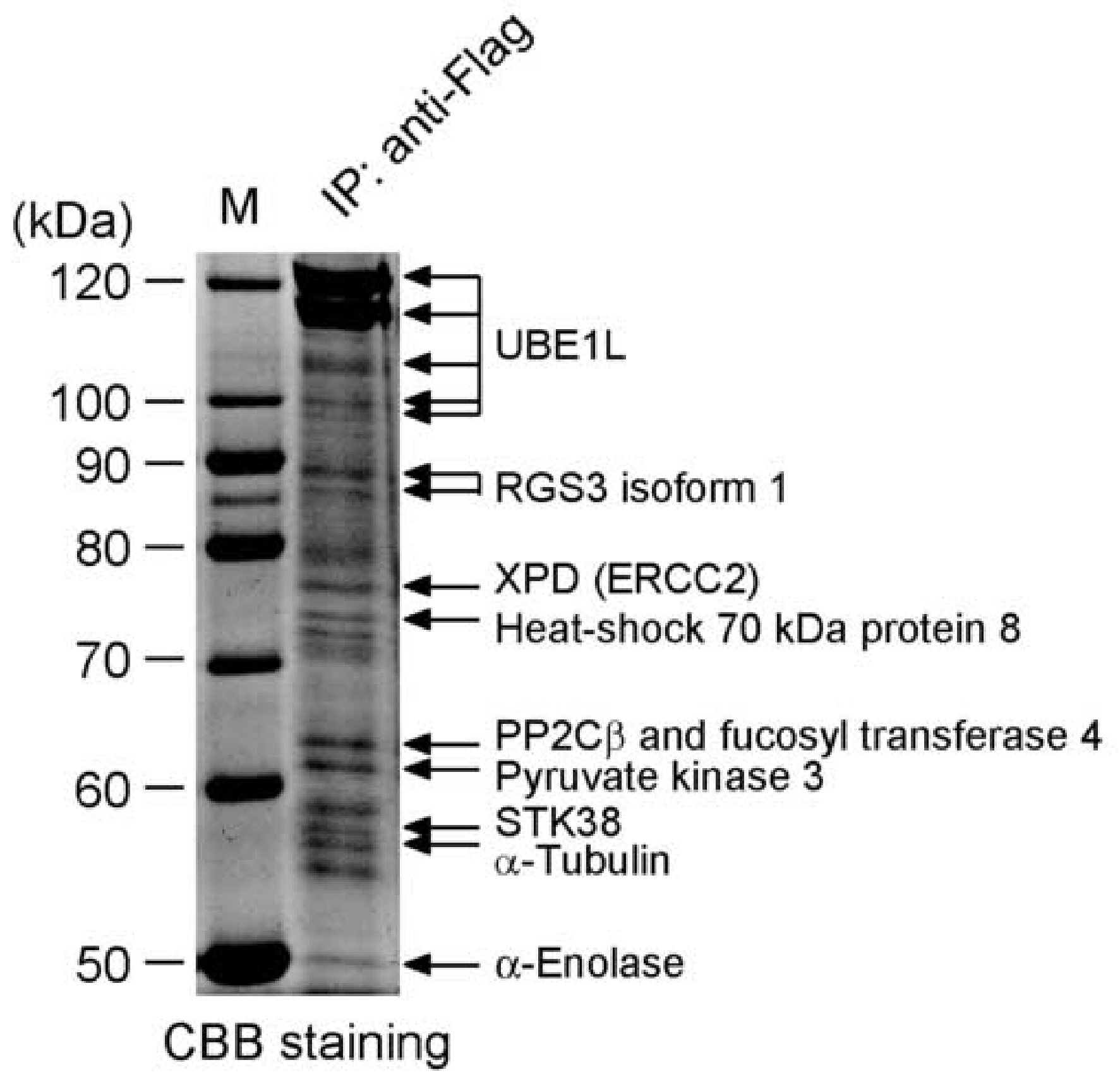


Fig. 2. Takeuchi et al.

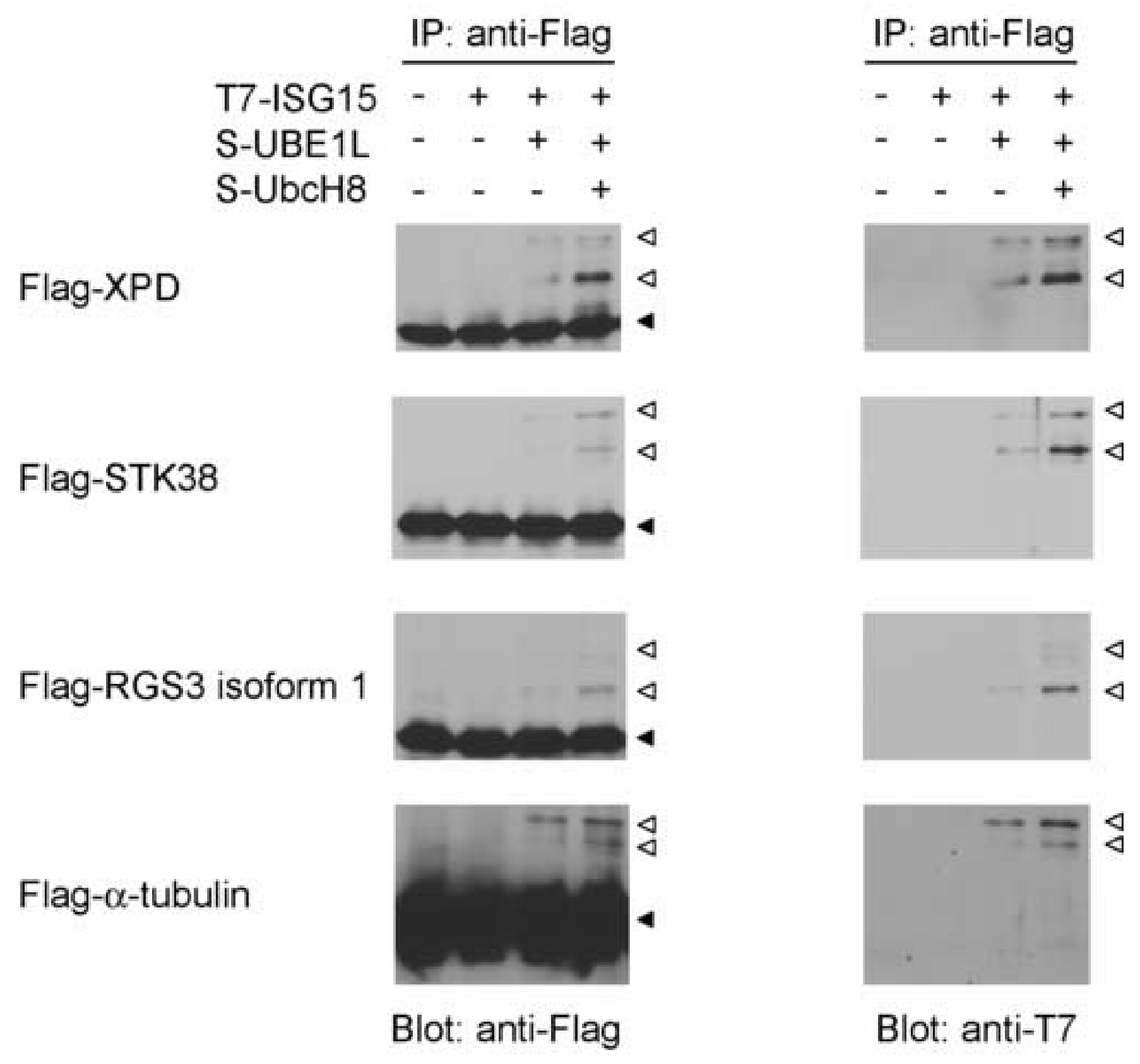




\section{Fig. 3. Takeuchi et al.}

T7-ISG15 -++++

S-UBE1L - -++++

S-UbcH8 - - -++

S-Efp - - $-\quad-\quad+$

S-Herc5 - - - - - +

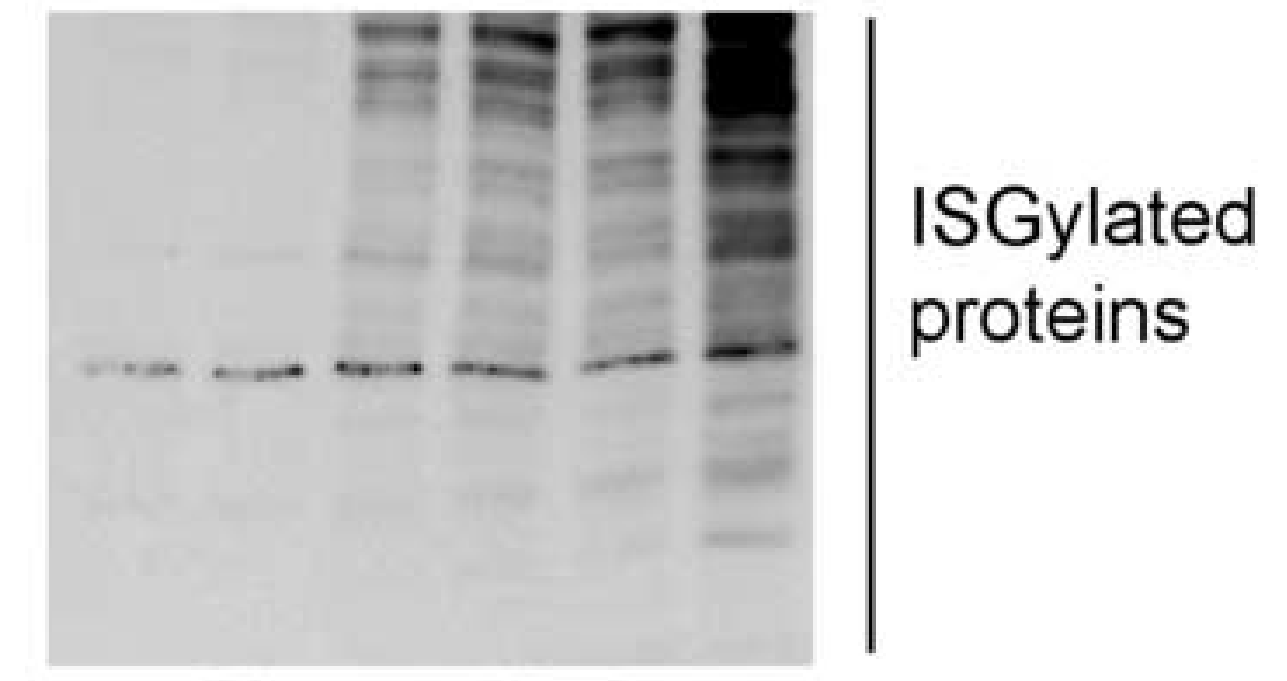

Blot: anti-T7
4 S-UBE1L
4 S-Efp
$4 \mathrm{~S}-\mathrm{UbcH} 8$

Blot: anti-S peptide 
Fig. 4. Takeuchi et al.

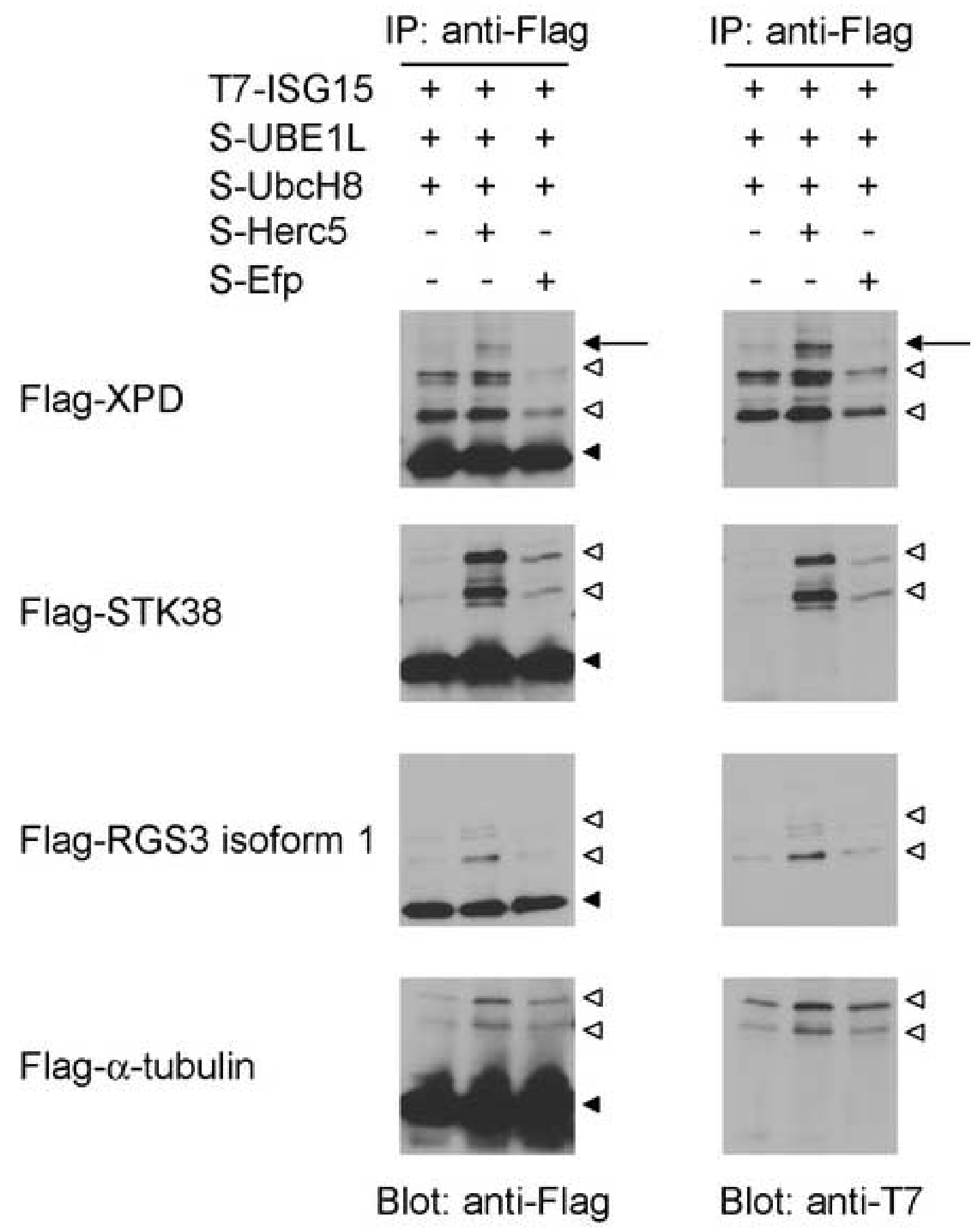


Fig. 5. Takeuchi et al.

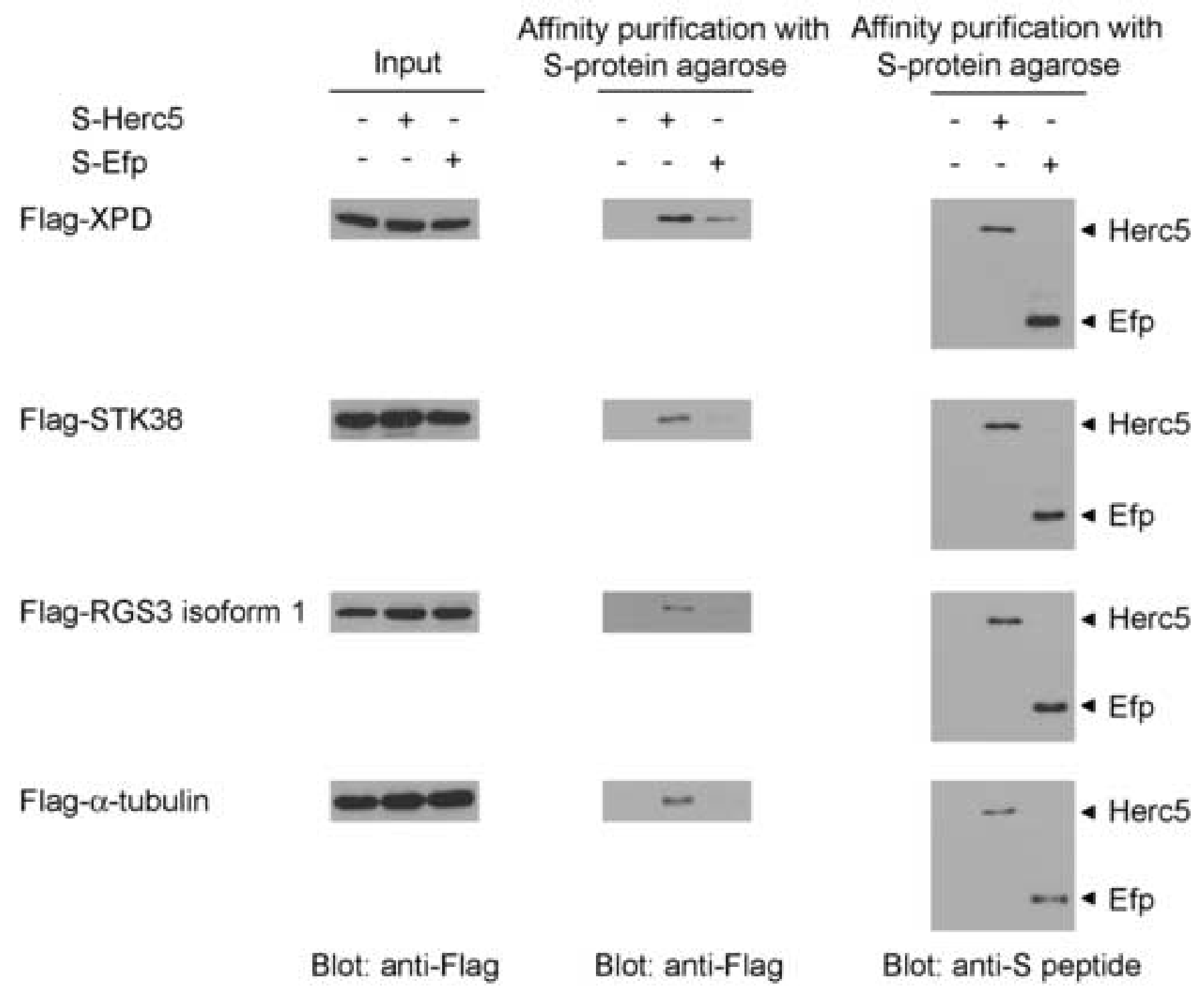


Table 1

Candidate target protein s for ISGylation identified in this study

Name

XPD (ERCC2) $)^{\mathrm{a}}$

$\operatorname{STK} 38^{\mathrm{a}}$

RGS3 isoform $1^{\mathrm{a}}$

$\alpha$-Tubulin ${ }^{\mathrm{a}}$

$\mathrm{PP} 2 \mathrm{C} \beta^{\mathrm{a}}$

Fucosyl transferase $4^{\mathrm{a}}$

UBE1 L ${ }^{b}$

Heat-shock $70 \mathrm{kDa}$ protein $8^{\mathrm{b}}$

Pyruvate kinase $3^{\mathrm{b}}$

$\alpha$-Enolase $\mathrm{e}^{\mathrm{b}}$
Function

DNA repair helicase

serine/threonine kinase

GTPase-activating protein

cytoskeleton

serine/threonine phosphatase

fucosyl transferase

E1 for IS Gylation

chaperone

phosphotransferase

phosphopyruvate hydratase

${ }^{a}$ Newly identified in this study.

${ }^{\mathrm{b}}$ Previously reported $[7,8]$. 